\title{
Prognostic factors in chronic hypersensitivity pneumonitis
}

\author{
Andrew W. Creamer $\mathbb{D}$ and Shaney L. Barratt \\ Affiliation: Bristol Interstitial Lung Disease Service, Southmead Hospital, North Bristol NHS Trust, Bristol, UK.
}

Correspondence: A.W. Creamer, Respiratory Dept, Southmead Hospital, North Bristol NHS Trust, Southmead Road, Bristol BS10 5NB, UK. E-mail: andrew.creameranhs.net

@ERSpublications

Hypersensitivity pneumonitis is a heterogeneous condition with variable prognosis. Clinical features including demographics and antigen exposure, and physiological, radiological and laboratory markers are associated with disease course and survival. http://bit.ly/2QtTYcv

Cite this article as: Creamer AW, Barratt SL. Prognostic factors in chronic hypersensitivity pneumonitis. Eur Respir Rev 2020; 29: 190167 [https://doi.org/10.1183/16000617.0167-2019].

ABSTRACT Hypersensitivity pneumonitis (HP) is an immunologically mediated lung disease resulting from exposure to inhaled environmental antigens. Prognosis is variable, with a subset of patients developing progressive fibrosis leading to respiratory failure and death. Therefore, there is an urgent need to identify factors which predict prognosis and survival in patients with HP. We undertook a narrative review of existing evidence to identify prognostic factors in patients with chronic HP. Patient demographics, smoking history, extent of antigen exposure and comorbidities all have reported associations with disease outcome, and physiological, radiological and laboratory markers have been shown to predict overall survival. While no single marker has been demonstrated to accurately and reliably predict prognosis, older age, more severe impairment of pulmonary function at baseline and established fibrosis on either biopsy or high-resolution computed tomography are consistently associated with worse survival. The vast majority of existing studies are retrospective, and this review identifies a need for prospective longitudinal studies with serial assessment of respiratory health to ascertain factors associated with nonfatal deterioration. Future developments, including the development of HP-specific composite scores may help further improve our ability to predict outcomes for individual patients.

\section{Introduction}

Hypersensitivity pneumonitis (HP) is an immunologically mediated lung disease resulting from exposure to inhaled environmental antigens. HP was first recognised as an occupational lung disease in those exposed to organic antigens and our understanding of the condition has become increasingly complex over the past decade.

A recently proposed classification uses clinical, radiological and pathological features to categorise cases into two distinct clusters $[1,2]$. The acute form presents with symptoms following antigen exposure, and is characterised by acute inflammatory infiltrates on imaging and bronchoalveolar lavage (BAL). Strict antigen avoidance, with or without a course of corticosteroids, would be expected to resolve symptoms and radiological infiltrates. The chronic fibrotic form of HP presents more insidiously, with symptoms gradually developing over several months, and is defined by the presence of established fibrosis on high-resolution computed tomography (HRCT) or histopathology. While there may be a degree of reversibility, established fibrosis will remain, and there is a risk of further progression. Longitudinal data [3], as well as scientific plausibility supports the progression of acute HP to the chronic form with the

This article has an editorial commentary https://doi.org/10.1183/16000617.0177-2020

Provenance: Submitted article, peer reviewed

Received: 01 Dec 2019 | Accepted after revision: 03 Jan 2020

Copyright CERS 2020. This article is open access and distributed under the terms of the Creative Commons Attribution Non-Commercial Licence 4.0. 
development of fibrosis, but the extent to which antigen avoidance (if identified) or immunomodulatory therapy can prevent this remains unknown.

Chronic HP presents a particular diagnostic challenge. While the disease definition implicates the presence of a causative environmental antigen, in many cases (in some series up to two-thirds) $[4,5]$ none can be identified. There are no established diagnostic criteria [6], with interstitial lung disease (ILD) multidisciplinary team (MDT) consensus being the gold standard for diagnosis. However, inter-MDT consensus has been recognised to be poor [7], and there remains difficulty in distinguishing chronic HP from other fibrotic ILDs, particularly idiopathic pulmonary fibrosis (IPF) [8].

IPF remains the most common and fatal ILD, and the condition has become well characterised over the past decade with recognised median life expectancy and prognostic scores developed, e.g. the GAP score (gender $(\mathrm{G})$, age (A), and two lung physiology variables (P) (forced vital capacity (FVC) and diffusing capacity of the lung for carbon monoxide $\left(D_{\mathrm{LCO}}\right)$ ) [9] and the DU BoIs score [10], as well as defined treatment pathways [11]. The course of disease in patients with HP can be heterogeneous with a proportion of patients developing a progressively fibrosing phenotype that shows similarities to IPF in terms of natural history and poor prognosis. A recent analysis of claims data from the USA found that only $58 \%$ of patients with fibrotic HP were still alive approximately 7 years after diagnosis [12]. Consequently, there is an urgent need to identify factors which predict prognosis and survival in patients with HP.

The primary aim of this concise review was to examine and summarise the current data on predictors of survival and disease progression in chronic HP.

\section{Search strategy and selection criteria}

We searched Pubmed and Google Scholar for articles published up to 29 July 2019 using the terms "hypersensitivity pneumonitis", "extrinsic alveolitis", "farmers lung" and synonyms, "pigeon/bird fanciers' lung" and synonyms and "prognosis", "disease course", "outcomes" and "survival". Relevant articles contained longitudinal follow-up data of a defined cohort of patients, including overall survival, transplant-free survival, and/or physiological parameters (including but not limited to spirometry, gas transfer and tests of exercise capacity). Articles resulting from these searches and relevant references were reviewed. Articles published or available in English were included.

\section{Prognostic factors in chronic cHP \\ Demographics}

The earliest studies into the epidemiology of HP, published in the 1960s to 1980s, described cohorts of individuals with occupational forms of the disease [13-15], with the earliest study identified (published in 1968) describing the outcomes of patients with farmers' lung [13]. More recent studies have used national surveys or insurance claims databases to attempt to cover all cases of the condition, including those where no inciting antigen can be identified.

Methodological differences may account for the variations in reported sex and age demographics, with a male sex typically more frequent in studies where an occupational definition was used [16, 17] while in studies using a hospital database, a national survey [18] or insurance data [12], the gender balance typically reflected a moderate female predominance of $55-60 \%[12,19-25]$.

Older age is widely reported to be associated with increased mortality. In a large retrospective study performed using an insurance claims database in the USA [12], 7498 cases of HP were identified over a 10 -year period. All-cause mortality rates were 115.9 per 1000 person-years in those aged $>65$ years $(95 \%$ CI 100.5-131.4), compared with 16 per 1000 person-years in those aged $45-54$ years (95\% CI 9.9-24.5) and 37.5 in those aged 55-64 years (95\% CI 28.6-48.3). However, mortality rates specifically due to HP could not be ascertained. Increasing age has also been associated with worse survival in multivariate survival analyses in cohorts from Spain ( $\mathrm{n}=160$, HR (95\% CI) 1.038 (1.012-1.064)) [21], Mexico [26] $(\mathrm{n}=78$, relative risk $(95 \%$ CI) $4.0(2.1-7.6))$, Brazil [27] $(\mathrm{n}=103$, HR $(95 \%$ CI) $1.10(1.03-1.18))$ and the UK (n=233, HR (95\% CI) 1.06 (1.03-1.08)) [22].

Several studies have reported that male sex is associated with worse survival [27]. The same insurance claims database [12] found a higher prevalence of HP in women (58\%), but higher mortality rates in men (all-cause mortality rate 63.9 per 1000 person-years in men (95\% CI 54.5-73.4) versus 40.1 per 1000 person-years in women (95\% CI 33.1-47.1)). Similarly, a Spanish cohort of 160 patients reported increased mortality with male sex (HR (95\% CI) 1.875 (1.167-2.956), p=0.009). Other cohort studies have found no difference in mortality between sexes [19, 22].

The influence of ethnicity on survival has only been explored in a small number of cohort studies. A 2016 study of 120 patients in the USA with chronic HP reported that transplant-free survival was greatest in 
non-Caucasian patients (HR (95\% CI) 8.42 (1.15-61.68), p=0.036) [20]; but non-Caucasian patients comprised only $17 \%$ of this cohort and the absolute numbers of patients that either died or underwent transplant were small.

\section{Antigen exposure}

The definition of HP implies the presence of a causative environmental antigen (inciting antigen (IA)), but in a substantial proportion of cases no IA could be identified; in some series in up to $63 \%$ of cases [5]. An essential initial management step in patients with HP is avoidance of the IA, and it is presumed that as persistent exposure can lead to disease progression, failure to identify (and remove) the IA could lead to worse outcomes. The impact of ongoing IA exposure on disease progression was investigated in a recent retrospective study of 202 patients in a Belgian cohort ( $n=109$ with established fibrosis; $n=93$ with no fibrosis) [28], in which the authors investigated the impact of both antigen avoidance and corticosteroid treatment on disease progression and survival. An IA was identified in $89 \%$ of cases with no fibrosis and $72 \%$ of cases with established fibrosis, and $72 \%$ of patients with an identified IA avoided further exposure (as a retrospective study there is no discussion of how avoidance was achieved or why the remaining cases continued to be exposed). In patients with no established fibrosis, IA avoidance was associated with an improvement in FVC of $0.92 \%$ per month, compared with a monthly FVC decline of $0.24 \%$ in those with ongoing IA exposure $(\mathrm{p}=0.016)$. However, no significant difference was seen with IA avoidance in patients with established fibrosis. There was no difference in overall survival with IA avoidance in either group.

Failure to identify an IA makes definitively avoiding further exposure impossible. The prognostic implication of identified versus unidentified IA in chronic fibrotic HP was investigated in a North American cohort of 142 patients [4]. An IA was identified in 47\% of cases, with this group having significantly longer median survival than in patients where no IA was identified, after adjusting for age, fibrosis, smoking history, FVC and $D_{\mathrm{LCO}}(8.75$ versus 4.88 years, $\mathrm{p}=0.047$ ). Small longitudinal studies have supported this hypothesis $[28,29]$, while others found no difference in survival in patients where no causative antigen can be identified [30, 31].

A wide range of IAs have been identified as causes of HP, with the most common being avian proteins, moulds and nontuberculous mycobacteria in metal-working fluid. It does not appear that different antigens are associated with different subsequent diseases courses, with a recent retrospective study of 155 patients with chronic HP reporting no difference in overall or transplant-free survival in patients with HP due to mould or avian antigens [32].

Other studies have investigated whether higher environmental levels or prolonged exposure to antigens are associated with disease progression. In a study of 23 patients with chronic bird-related HP, levels of avian antigen in household dust were measured [33]. Patients had lung function tests repeated at 1 year after study initiation. Patients whose condition showed deterioration $(n=11)$ had significantly higher levels of avian antigen in household dust samples compared with those who showed stability $(n=12)$. A subsequent study in a cohort of 14 patients showed similar results [34], with a higher rate of annual decline in FVC in patients with a high level of avian antigen in home dust compared with those with a lower level $(342 \pm 107$ versus $116 \pm 126 \mathrm{~mL}, \mathrm{p}=0.014)$. However, the relationship between levels of antigen exposure and disease progression is not clear, with cohorts of patients with farmer's lung [14,35] and pigeon breeders [36] demonstrating disease stability or improvement regardless of whether they maintained avoidance or not.

There are a number of plausible explanations for the conflicting data on the impact of antigen exposure on disease course in patients with HP. One possibility proposed by the authors of a study in a population of bird keepers is the difficulty in confirming whether true antigen avoidance has been achieved [36]; inadequate protective equipment, difficulty in changing workplace practices or persisting antigen in the environment (e.g. bird feathers or droppings remaining after bird removal) could all cause ongoing exposure. Recognising that individual workers with the same occupational exposure may not develop any disease, other groups [4] have proposed that innate differences between individual's immune responses to inhaled IA alter the risk of the development and progression of HP, even between individuals with identical environmental exposures. Finally, the extent to which an identified IA is necessary for a diagnosis of HP to be made remains controversial [6], and the inter-MDT agreement for diagnosis of HP has been recognised as being poor [7]. This raises the possibility that some of the studies containing patients with unidentified aetiological antigen may include misdiagnoses of other fibrotic lung diseases as chronic HP.

\section{Smoking}

There is a significant body of evidence that suggests smoking is associated with worse overall survival in patients with HP, although this correlation is not universally reported in all cohorts. 
The largest study describing a link between smoking and prognosis in patients with HP (177 in a US cohort) [29] found smoking was associated with all-cause mortality in multivariate analysis (HR (95\% CI) 2.68 (1.26-5.73), $\mathrm{p}=0.01$ ) and other studies have reported similar findings [4, 37]. These findings are not replicated in all cohort studies, with studies in a US population $(n=119)$ [3] and a Spanish population $(n=160)$ [21] reporting no correlation between smoking status and survival. As with many of these studies, most of the cohort remained alive at the end of the follow-up period with low overall numbers of deaths observed in either group, limiting the power of statistical comparison. Furthermore, the use of all-cause mortality means it is difficult to ascertain what impact smoking has on progression of HP, and what contribution to worse survival in smokers is due to other smoking-related complications such as cancer or cardiovascular disease.

Historical data suggest that while acute HP may be more common in nonsmokers than current smokers [38], the development of fibrosis is more common in smokers. A 1995 retrospective study of 43 individuals with farmers' lung in Japan compared disease presentation and prognosis in 12 smokers to 31 never-smokers [37] and reported that fibrosis was more common in smokers (present in 33.3\% of smokers compared with $6.5 \%$ of never-smokers).

\section{Comorbidities}

We identified four papers specifically investigating the impact of comorbidities on prognosis in HP.

Group 3 pulmonary hypertension (PH) develops due to lung disease and/or hypoxia, and can complicate ILD. The prevalence in patients with IPF is estimated to be 30-50\% [39]. A retrospective study of 120 patients with HP (83 with chronic HP) detected PH (defined in the study as systolic pulmonary artery pressure (sPAP) $\geqslant 50 \mathrm{mmHg}$ on doppler echocardiography) in 14 (19\%) patients [40]. The presence of $\mathrm{PH}$ was associated with significantly worse survival over the study period (HR (95\% CI) 4.00 (1.49-10.73), $\mathrm{p}=0.006$ ), a significantly stronger predictor of survival than FVC (HR (95\% CI) 0.97 (0.95-0.99), p0.027) and $D_{\mathrm{LCO}}(\mathrm{HR}(95 \% \mathrm{CI}) 0.96(0.92-1.0), \mathrm{p}=0.063)$. This mirrors findings in patients with IPF, where sPAP $\geqslant 50 \mathrm{mmHg}$ significantly worsened survival $[39,41]$. As a simple noninvasive investigation and strong predictive factor for survival (albeit in a retrospective study with small numbers), doppler echocardiography to assess for PH deserves further investigation as a prognostic tool in patients with HP.

The presence of coexistent lung pathologies is recognised in patients with fibrotic ILDs, and combined pulmonary fibrosis and emphysema (CPFE) is considered a distinct clinical syndrome [42]. A recent study of 233 patients with HP in a UK population reported emphysema in $30 \%$ of patients with HP, including in $23 \%$ of never-smokers [22]. Patients with emphysema demonstrated a typical CPFE profile of artificially preserved lung volumes with a disproportionate reduction in transfer factor of the lung for carbon monoxide, but the study found that there was no significant difference in survival in HP patients with emphysema compared with those without.

Pleuroparenchymal fibroelastosis (PPFE) is an increasingly recognised ILD, and while PPFE can develop idiopathically [43], it has also been reported to develop in association with other ILDs [44]. A 2018 study of 233 patients with HP found evidence of PPFE in 40\% of patients with HP, and PPFE of "marked" severity in $23 \%$ [22]. Although not a significant predictor of mortality in a univariable Cox mortality analysis, in a multivariable model adjusted for patient age, sex, smoking status and ILD extent, marker PPFE independently predicted mortality (HR (95\% CI) 1.97 (1.16-3.33), p=0.01).

Given the immune-mediated pathogenesis of HP, some researchers have hypothesised that the presence of other autoimmune conditions may be associated with disease course. A 2016 study in the USA of 120 patients with chronic fibrotic HP found $15 \%$ had evidence of autoimmunity (either a concurrent autoimmune disease or autoimmune features of a connective tissue disease) [20]. On unadjusted comparison of chronic fibrotic HP with autoimmune features to chronic fibrotic HP without autoimmune features, there was no significant difference in overall survival. However, when adjusted for age, ethnicity, sex, body mass index, oxygen therapy and disease severity, the presence of autoimmune features was identified as an independent factor predicting mortality or transplant (multivariate Cox regression analysis, HR (95\% CI) 4.45 (1.43-13.88), p=0.01). Interestingly, despite predicting worse survival, there was no difference in rate of FVC decline between patients with and without autoimmune features.

\section{Lung physiology}

Baseline lung function tests have prognostic value in patients with IPF, reflected in the GAP index tool [9] in which FVC \% predicted and predicted $D_{\mathrm{LCO}}$ comprised two of the four variables. Baseline lung physiology has also been widely shown to predict survival in HP cohorts [3, 19, 21, 25, 45], with a lower FVC $\%$ pred, total lung capacity and $D_{\mathrm{LCO}}$ associated with increased mortality. 
Serial lung function tests have been reported to predict survival. In a Brazilian cohort of 112 patients, those with $\mathrm{a} \geqslant 10 \%$ decline in predicted FVC after 6-12 months had a significantly increased all-cause mortality, with median (95\% CI) survival of 53 (37-69) months compared with median (95\% CI) survival of $139(66-212)$ months $(\mathrm{p}=0.007)$ in those with stable FVC [25].

\section{Radiology}

In current clinical practice, the distinction between the acute and chronic forms of HP is increasingly made on the basis of clinical, radiological and pathological features, rather than just chronicity of symptoms $[1,2]$. The presence of established fibrosis on HRCT is integral to this definition. Considering the extent to which radiological features of fibrosis alter disease course in patients with HP is, therefore, analogous to comparing the natural histories of the acute and chronic forms of the condition. Multiple studies have shown that the presence of fibrosis, regardless of whether diagnosed on histopathology or radiology, gives a worse survival than HP without established fibrosis [19, 27, 29-31, 46, 47]. Conversely, ground-glass opacification, a feature indicating acute inflammatory infiltrates [46] and air trapping, and mosaic attenuation (radiological features of small airway inflammation) have been associated with improved survival $[27,47]$.

SALISBURY et al. [48] phenotyped 117 patients with HP into three groups based on CT appearances: no fibrosis, non-honeycomb fibrosis and honeycomb fibrosis. The patients with no fibrosis demonstrated improving lung function (FVC \% pred) over time and had the longest event-free median survival at $>14.73$ years. Patients with established fibrosis showed deterioration in lung function, with patients with non-honeycomb fibrosis having a median survival of 7.95 years (compared with 5.2 years in a matched cohort of patients with IPF). Patients with HP and honeycomb fibrosis had poor survival (2.76 years), which was equivalent to that of a matched cohort of patients with IPF (2.81 years). This indicates the importance of establishing a correct diagnosis in patients with non-honeycomb fibrosis, as there is a significant survival difference between HP and IPF at this stage; by the time honeycombing is present, prognosis is uniformly poor across the two conditions.

A similar approach was undertaken by Mooney et al. [29], where a semi-quantitative analysis of extent of fibrosis was used to produce a CT fibrosis score. CT fibrosis score was independently associated with mortality and/or transplantation (HR 1.35, $\mathrm{p}<0.01$ ). When compared with a cohort with IPF, survival was better in patients with HP when matched for extent of radiographic fibrosis. A further study in 92 patients with chronic HP found that distinct HRCT patterns, particularly severity of traction bronchiectasis, but also the presence and extent of micro- and macro-cystic honeycombing and global ILD extent independently predicted mortality [49]. The study reported that these radiological markers more accurately predicted mortality than lung physiology. However, the recognised variability in CT reporting means that novel radiological biomarkers such as these may need to wait until low cost and validated systems are widely available before they can enter routine clinical use [49].

More recently, researchers have investigated how automated analysis of CT can generate prognostic markers in chronic HP. CALIPER (Computer-Aided Lung Informatics for Pathology Evaluation and Rating) is a software tool that can perform sophisticated quantitative CT evaluation. In a cohort of 116 patients with MDT diagnoses of chronic HP, visual/CALIPER measures of reticulation, honeycombing and traction bronchiectasis, as well as CALIPER ILD extent, were predictive of mortality on univariate analysis [45]. A further radiological feature, pulmonary vessel volume (PVV) was specifically identified as a prognostic marker, independent of physiological measurements. Specifically, a PVV $>6.5 \%$ of the total lung volume (identified in $17 \%$ of the total HP cohort) was found to predict an aggressive disease course, with median survival nearly identical to that of a matched IPF cohort. A second study by the same group found that use of CALIPER analysis was a stronger predictor of mortality than visual CT analysis, and that the prognostic accuracy of the ILD-GAP model in chronic HP was improved when combined with quantitative CT analysis [70]. While the use of computer-aided CT analysis offers the potential of a noninvasive prognostic marker, such findings are yet to be replicated in an independent cohort, and use in clinical practice remains to be determined.

\section{Histopathology}

As with radiology, presence of established fibrosis on biopsy is an indicator of worse survival [3, 27]. In a study of 72 patients in the USA, all with biopsy-confirmed HP, the presence of fibrosis was predictive of increased mortality in age-adjusted analysis (HR (95\% CI) 6.01 (1.68-21.45), p=0.006). In this cohort, the mean survival of patients with established fibrosis was 7.1 years, which remains considerably longer than that expected with IPF.

Chronic HP has characteristic histological features including poorly formed granulomas, airway-centred inflammation and bridging fibrosis. However, it is recognised that other histological patterns including 
cellular nonspecific interstitial pneumonia (NSIP) or fibrotic NSIP and usual interstitial pneumonia (UIP), or features associated with other ILDs such as fibroblastic foci (a hallmark of UIP and IPF) may also be present. A number of retrospective studies have investigated the prognostic impact of differing pathological subtypes (as fitness to undergo surgical lung biopsy was a de facto inclusion criteria, there is risk of selection bias in these studies) [3, 26, 27, 50-53]. The presence of a UIP pattern of fibrosis has consistently been identified as a poor prognostic marker $[3,50,52,53]$, in some cases giving a mean survival similar to that seen in patients with IPF (2.8 years) [50]. Both a fibrotic NSIP pattern and bronchiolocentric pattern of fibrosis have also been associated with worse outcomes when compared with "typical" HP patterns [3, 50, 52]. A study of 16 patients in Japan, all with a diagnosis of chronic bird-related HP and a UIP pattern of fibrosis on surgical lung biopsy, found that higher numbers of fibrotic foci were associated with a worse prognosis than a UIP pattern with lower numbers of fibrotic foci [51]. Patients with high numbers of fibrotic foci experienced a disease course similar to that seen in patients with IPF (median survival 27 months from date of biopsy). Although the above studies demonstrate a correlation with histopathological features and disease course, there is a significant mortality associated with surgical lung biopsy for the diagnosis of ILD (2.4\% 30-day mortality) [54]. Guidelines are therefore increasingly moving away from undertaking numerous biopsies, so it is unlikely that these findings will be useable for prognostic information in routine clinical practice.

\section{Genetics}

Several genes have been investigated as predictors of progression in fibrotic ILDs. In IPF, genome-wide association studies (GWAS) have identified several polymorphisms that are associated with the condition, including genes involved in telomere maintenance, mucin production and the innate immune response. The MUC5B minor allele polymorphism, encoding a mucin gene, has been shown to be associated with a substantially higher risk of IPF, but also with improved survival in patients with the condition [55]. Polymorphisms in TOLLIP, a regulator of innate immune responses mediated via Toll-like receptors, have also been shown to alter risk of developing the condition and survival in those affected [56].

We identified two studies which investigated genetic factors in HP. LeY et al. [24] measured two single-nucleotide polymorphisms known to be associated with IPF (in MUC5B and TOLLIP) in two cohorts of patients with chronic HP ( $n=145$ and $n=72)$. In chronic HP, the MUC5B rs35705950 single-nucleotide polymorphism (SNP) was associated with moderate-severe fibrosis and traction bronchiectasis on CT. Unlike in patients with IPF where this SNP is associated with improved survival, in chronic HP the presence of the minor allele had no significant association with survival, although trended towards worse mortality (adjusted HR (95\% CI) $2.01(0.97-4.20), \mathrm{p}=0.063)$. There was no association for TOLLIP polymorphisms with predisposition, fibrosis or survival. The same study also investigated telomere length in peripheral blood leukocytes. In patients with chronic HP, shorter telomere length was associated with worse survival, as well as more severe fibrosis on CT; indeed, with a radiological and histopathological picture similar to IPF.

The potential role of telomere dysfunction in chronic HP was further investigated in a more recent study by the same group [57], which investigated the frequency and effect of mutations in telomere maintenance genes on survival in chronic HP. Variants in telomere-related genes were identified in 16 out of 144 patients in the discovery cohort, and 17 out of 209 in the replication cohort. The presence of these alleles significantly reduced transplant-free survival (adjusted HR (95\% CI) 3.73 (1.92-7.28) in discovery cohort; HR (95\% CI) 2.72 (CI 1.26-5.88) in validation cohort).

These results demonstrate the impact of genetic factors in the prognosis and clinical course of HP. By focusing on genes already known to be involved in IPF, it is perhaps unsurprising that their effect in HP is to give a more IPF-like disease, with more severe fibrosis and a worse prognosis. The authors acknowledge that, given the recognised risk of misclassifying IPF and chronic HP, it may be that some of the patients may be misdiagnosed. However, by ensuring a multidisciplinary approach to diagnosis, the authors were confident that this risk was mitigated. It will be of interest if future GWAS in chronic HP identify significant polymorphisms in genes distinct from those seen in IPF.

\section{Acute exacerbation}

An acute exacerbation of IPF is defined as an acute, significant respiratory deterioration of unidentified cause, with criteria including the presence of new radiological abnormalities on HRCT and the exclusion of alternative aetiologies [58]. Prognosis is poor, with in-hospital mortality reported as 50\% [59]. While initially identified in patients with IPF, it is increasingly recognised as a feature of other fibrotic ILDs [60]. We identified three papers investigating acute exacerbations in chronic HP. A retrospective study in a US cohort identified four cases of acute exacerbations in chronic HP [61] (who met criteria from the consensus statement from the IPF clinical research network on acute exacerbations in patients with IPF 
[58]). Three of these patients died within or shortly after the admission, while the fourth underwent urgent bilateral lung transplantation.

A retrospective study of 100 patients with chronic avian-related HP over a 13-year period identified 14 patients who experienced acute exacerbations in chronic HP [62]. All of these patients were found to have a UIP pattern on either radiology or biopsy, and had a neutrophil predominance with lower lymphocytes on BAL than patients who did not exacerbate. A further study of 56 patients with chronic avian-related HP who underwent extensive investigations including BAL and surgical lung biopsy at baseline identified subsequent acute exacerbations in 14 (25\%) patients in the cohort [63]. There was no difference in age between those that experienced acute exacerbations in chronic HP and no significant difference in smoking status. Again, a UIP pattern on biopsy was more prevalent in patients who experienced acute exacerbations in chronic HP (12 out of 14), although a significant number of patients who did not experience acute exacerbations in chronic HP also had UIP (19 out of 42). The authors reported that serum (but not serum and BAL fluid) levels of CCL17, a Th2 chemokine, were higher in patients who subsequently developed acute exacerbations in chronic HP, and suggested a role for its use a predictor of future acute exacerbations. However, as a significant number of other cytokines and biomarkers were also investigated, further studies are required to ascertain its true significance. $12(86 \%)$ of the 14 patients died during the acute exacerbations.

\section{Serum and BAL biomarkers}

Given the risks associated with surgical lung biopsy and the inter-observer variability in interpreting HRCT images, there is significant interest in the development of serum and BAL fluid biomarkers to aid in prognostication in fibrotic ILDs. In IPF, identified serum biomarkers include epithelial proteins CA19-9, CA-125, MMP-7 and SPD64, and KL-6, a glycoprotein expressed on Type 2 pneumocytes [64]. Although these have been associated with higher risk of progression in patients with IPF, at the time of writing their use is not recommended for routine use in the American Thoracic Society/European Respiratory Society guidelines [65].

A summary of studies investigating serum biomarkers of prognosis in patients with HP is given in table 1. Reflecting the complex pathogenesis of HP, biomarkers include epithelial and extracellular matrix proteins (SP-D and periostin, respectively), glycoproteins expressed on pneumocytes (KL-6), proteins involved in the immune response (CCL-17) and chitinases (YKL-40). Although correlations with disease progression and survival were reported with many of the serum biomarkers, none of these studies have been subsequently validated in prospective cohort studies.

A more readily available potential biomarker is the extent of lymphocytosis on BAL, with a recent DELPHI survey consensus that lymphocytosis of $>40 \%$ was an "important" diagnostic factor for the condition [6]. A recent prospective cohort study found no correlation between initial BAL lymphocytosis and disease progression [69].

\begin{tabular}{|c|c|c|c|c|c|c|}
\hline $\begin{array}{l}\text { First author } \\
\text { [ref.] }\end{array}$ & $\begin{array}{l}\text { Study } \\
\text { location }\end{array}$ & Population & $\begin{array}{c}\text { Subjects } \\
n\end{array}$ & $\begin{array}{l}\text { Biomarker of } \\
\text { interest }\end{array}$ & $\begin{array}{l}\text { Primary } \\
\text { outcome }\end{array}$ & Results \\
\hline Nukui [66] & Japan & $\begin{array}{c}\text { Chronic } \\
\text { bird-related HP }\end{array}$ & 63 & Periostin & $\begin{array}{l}\text { All-cause } \\
\text { mortality }\end{array}$ & $\begin{array}{l}\text { Higher serum periostin levels are associated with worse } \\
\text { survival (periostin }>92.5 \mathrm{ng} \cdot \mathrm{mL}^{-1} \text { mean }(95 \% \mathrm{Cl} \text { ) survival } \\
34.8(25.2-44.5) \text { months; perisostin }<92.5 \mathrm{ng} \cdot \mathrm{mL}^{-1} \text { mean } \\
\text { (95\% CI) survival } 65.0(46.0-84.1) \text { months) }\end{array}$ \\
\hline Long [67] & Germany & $\begin{array}{l}\text { HP }(11 \text { acute/ } \\
\text { subacute, } 61 \\
\text { chronic) }\end{array}$ & 72 & $\begin{array}{l}\text { YKL-40 (serum } \\
\text { and BALF) }\end{array}$ & $\begin{array}{l}\text { Disease } \\
\text { progression } \\
\text { All-cause } \\
\text { mortality }\end{array}$ & $\begin{array}{l}\text { Higher serum YKL-40 levels are associated with disease } \\
\text { progression (cut-off } 119 \mathrm{ng} \cdot \mathrm{mL}^{-1} \mathrm{HR} \text { 6.567; } \mathrm{p}<0.001 \text { ) } \\
\text { Higher serum YKL-40 levels } \\
\text { are associated with worse survival lcut-off } 150 \mathrm{ng} \cdot \mathrm{mL}^{-1} \text {; } \\
\text { HR 9.989; } \mathrm{p}<0.001 \text { ) }\end{array}$ \\
\hline MIYAZAKAI [63] & Japan & $\begin{array}{c}\text { Chronic } \\
\text { bird-related HP }\end{array}$ & 56 & CCL-17 & $\begin{array}{l}\text { Incidence of } \\
\text { acute } \\
\text { exacerbation }\end{array}$ & $\begin{array}{c}\text { Higher serum CCL-17 levels are associated with } \\
\text { increased incidence of acute exacerbations ( } 2 \text {-year } \\
\text { incidence of acute exacerbations with baseline } \mathrm{CCL}-17 \\
>285 \mathrm{pg} \cdot \mathrm{mL}-130.1 \% \text {, baseline } \mathrm{CCL}-17<285 \mathrm{pg} \cdot \mathrm{mL}-1 \\
3.3 \% \text { ) }\end{array}$ \\
\hline JANSSEN [68] ${ }^{\#}$ & Netherlands & Bird-related HP & 49 & $\begin{array}{c}\mathrm{KL}-6 \text { and } \\
\text { SP-D }\end{array}$ & Change in $D_{\mathrm{LCO}}$ & Not available \\
\hline
\end{tabular}

HP: hypersensitivity pneumonitis; BALF: bronchoalveolar lavage fluid; $D_{\mathrm{LCO}}$ : diffusing capacity of the lung for carbon monoxide, CCL-17: C-C chemokine ligand 17, KL-6: Krebs von den lungen 6, SP-D: surfactant protein-D. \# : unable to access full paper. 


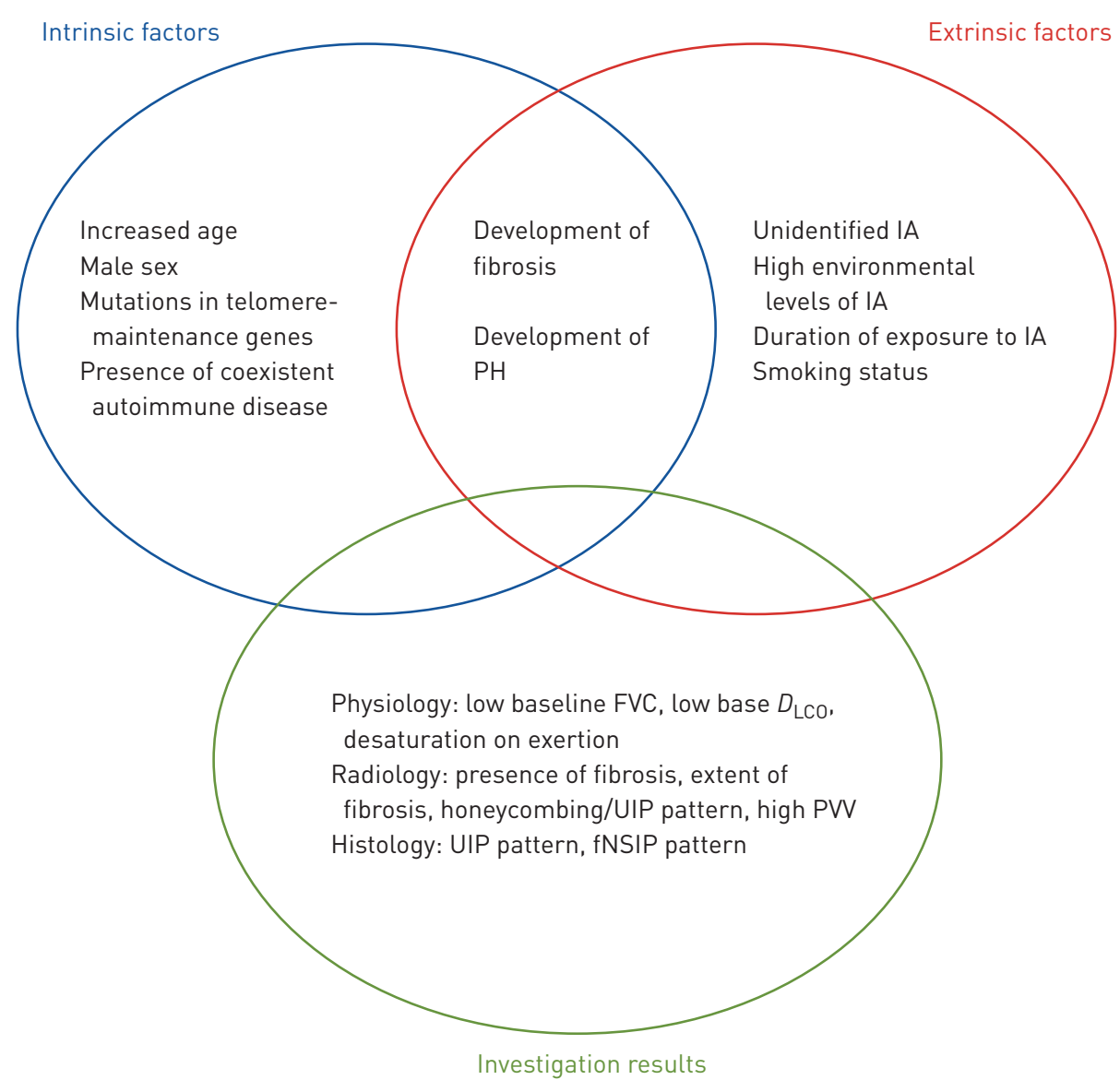

FIGURE 1 Summary of factors associated with increased mortality in hypersensitivity pneumonitis. $\mathrm{PH}$ : pulmonary hypertension; IA: inciting antigen; FVC: forced vital capacity; $D_{\text {LCO }}$ : diffusing capacity of the lung for carbon monoxide; UIP: usual interstitial pneumonia; PVV: pulmonary vessel volume; fNSIP: fibrotic nonspecific interstitial pneumonia.

\section{Discussion}

HP is a complex, heterogeneous condition. The disease course can be plausibly influenced by a large number of variables (patient demographics and genetics, chronicity and nature of antigen exposure, smoking, comorbidities), with an equally large number of clinical parameters used to assess patients (figure 1). It is therefore perhaps not surprising that identifying discrete factors that reliably and powerfully predict prognosis in this condition is challenging. Furthermore, there are significant limitations with the literature. The relatively long median survival means that in many published studies the majority of patients are still alive at time of censor; conclusions on factors which affect survival are therefore drawn from small numbers of patients. The lack of defined diagnostic criteria also means significant heterogeneity in cohorts studied; while a UIP pattern of fibrosis and no identified IA may indeed signify a poor prognosis in chronic HP, many clinicians would instead suggest that this indicates an alternative diagnosis [6]. Furthermore, for the purposes of this review both acute and chronic forms of HP were considered, with individual factors (presence of fibrosis, duration of antigen exposure) assessed as independent factors.

Nevertheless, there are some conclusions we can draw. Older patients and those with more severe impairment of lung physiology at baseline have higher mortality. Male sex and an unidentified precipitating antigen may be associated with mortality, although this association is not seen across all studies. The presence of comorbidities, particularly $\mathrm{PH}$, autoimmune conditions and PPFE also worsens prognosis. The presence of fibrosis, whether identified on CT or biopsy is consistently associated with increased mortality, while certain radiological features, particularly honeycomb fibrosis, more extensive disease and PVV have been all been identified as predicting mortality.

While the reported median survival of 7 to 9 years [24, 51, 70, 71], even with established fibrosis, is significantly longer than that seen in patients with IPF, multiple studies have identified a phenotype of chronic HP which experiences a significantly worse prognosis comparable to that of IPF $[48,72]$. Given that 
the identified predictors of this poor outcome were a UIP pattern of fibrosis and higher numbers of fibrotic foci, both features of IPF, the possibility of a misdiagnosis in these patients must be raised, but nevertheless their presence in any patient with fibrotic lung disease should alert the physician to a higher risk of mortality.

Other factors, such as MUC5B polymorphisms, shorter telomere length and computer-interpreted indices on HRCT are unlikely to be of routine use to clinicians at present, but offer exciting potential markers for the future. However, the hunt for novel biomarkers should not be the sole aim of future research into the condition; multicentre prospective studies, adequately powered and with sufficient follow-up to ascertain overall survival and with serial assessments of respiratory health to assess non-fatal deterioration are vital for improving our understanding of this condition.

\section{Future directions}

Composite scores, which combine factors to more robustly predict outcomes are widely used in respiratory medicine. The GAP score was initially developed to predict survival in patients with IPF, but has since been shown to also apply in other fibrotic ILDs, including rheumatoid arthritis and systemic sclerosis-associated ILD. At the time of writing, the GAP score has not been formally validated in patients with HP, but it is likely that a similar composite score would be of more powerful predictive value than any of the individual factors discussed in this article.

An alternative approach that has been recently proposed is to move away from current diagnostic labels of fibrotic ILDs altogether, and instead classify patients according to phenotypic features [73]. In this study, the authors took patients with IPF, HP, connective tissue disease-associated-ILD and interstitial pneumonia with autoimmune features, and used clinical and radiological factors (including race, body mass index, cigarette smoking and pulmonary artery diameter) to categorise them into one of four phenotypic clusters. These clusters were found to be more strongly predictive of both transplant-free survival and monthly FVC decline then the traditional diagnostic labels. While this study has not yet led to widespread adoption of this approach, it suggests that disease course may be better predicted by identifying key phenotypic features rather than diagnostic labels.

Although the majority of existing literature on HP comes from retrospective, often single-centre cohorts, there are a number of recent and current multicentre studies, including the INBUILD trial [74] and the INJUSTIS registry [75], collecting prospective data on HP (as well as other fibrotic ILDs). These studies in well characterised patient cohorts offer the potential to significantly improve our understanding of this condition and shape the way we manage progressive disease.

\section{Conclusions}

Physicians treating a patient with HP should recognise the complex interplay of innate and external factors that affect the outcome of the condition. In the absence of a simple prognostic tool, a thorough assessment of antigen exposure, comorbidities and respiratory function should be undertaken in all patients. Older age, more severe respiratory impairment at baseline, $\mathrm{PH}$ (sPAP $>50 \mathrm{mmHg}$ ) and established fibrosis, particularly UIP pattern with established honeycombing should alert the physician to a worse prognosis.

Future research should aim to identify novel biomarkers for prognosis, which may be radiological, physiological or biochemical. Building on this review, meta-analyses to answer specific questions are needed. Finally, there is a need for longitudinal studies with serial assessment of respiratory health to ascertain factors for nonfatal deterioration; multicentre and international collaboration efforts are essential to achieve this.

Conflict of interest: A.W. Creamer has nothing to disclose. S.L. Barratt has nothing to disclose.

\section{References}

1 Vasakova M, Morell F, Walsh S, et al. Hypersensitivity pneumonitis: perspectives in diagnosis and management. Am J Respir Crit Care Med 2017; 196: 680-689.

2 Salisbury ML, Myers JL, Belloli EA, et al. Diagnosis and treatment of fibrotic hypersensitivity pneumonia. Where we stand and where we need to go. Am J Respir Crit Care Med 2017; 196: 690-699.

3 Wang P, Jones KD, Urisman A, et al. Pathologic findings and prognosis in a large prospective cohort of chronic hypersensitivity pneumonitis. Chest 2017; 152: 502-509.

4 Fernández Pérez ER, Swigris JJ, Forssén AV, et al. Identifying an inciting antigen is associated with improved survival in patients with chronic hypersensitivity pneumonitis. Chest 2013; 144: 1644-1651.

5 Mokhlis J, Robertson AS, Moore VC, et al. Distribution of occupational and non-occupational causes in hypersensitivity pneumonitis diagnosed by an interstitial lung disease expert panel. Thorax 2017; 72: A64-A65.

6 Morisset J, Johannson KA, Jones KD, et al. Identification of diagnostic criteria for chronic hypersensitivity pneumonitis: an International Modified Delphi Survey. Am J Respir Crit Care Med 2018; 197: 1036-1044. 
$7 \quad$ Walsh SLF, Wells AU, Desai SR, et al. Multicentre evaluation of multidisciplinary team meeting agreement on diagnosis in diffuse parenchymal lung disease: a case-cohort study. Lancet Respir Med 2016; 4: 557-565.

8 Morell F, Villar A, Montero MÁ, et al. Chronic hypersensitivity pneumonitis in patients diagnosed with idiopathic pulmonary fibrosis: a prospective case-cohort study. Lancet Respir Med 2013; 1: 685-694.

9 Ley B, Ryerson CJ, Vittinghoff E, et al. A multidimensional index and staging system for idiopathic pulmonary fibrosis. Ann Intern Med 2012; 156: 684-691.

10 du Bois RM, Weycker D, Albera C, et al. Ascertainment of individual risk of mortality for patients with idiopathic pulmonary fibrosis. Am J Respir Crit Care Med 2011; 184: 459-466.

11 Raghu G, Rochwerg B, Zhang Y, et al. An Official ATS/ERS/JRS/ALAT Clinical Practice Guideline: treatment of idiopathic pulmonary fibrosis. An update of the 2011 Clinical Practice Guideline. Am J Respir Crit Care Med 2015; 192: e3-19.

12 Fernandez Perez ER, Kong AM, Raimundo K, et al. Epidemiology of hypersensitivity pneumonitis among an insured population in the United States: a claims-based cohort analysis. Ann Am Thorac Soc 2018; 15: 460-469.

13 Barbee RA, Callies Q, Dickie HA, et al. The long-term prognosis in farmer's lung. Am Rev Respir Dis 1968; 97: $223-231$.

14 Cormier Y, Belanger J. Long-term physiologic outcome after acute farmer's lung. Chest 1985; 87: 796-800.

15 Braun SR, doPico GA, Tsiatis A, et al. Farmer's lung disease: long-term clinical and physiologic outcome. Am Rev Respir Dis 1979; 119: 185-191.

16 Barber CM, Wiggans RE, Carder M, et al. Epidemiology of occupational hypersensitivity pneumonitis; reports from the SWORD scheme in the UK from 1996 to 2015. Occup Environ Med 2017; 74: 528-530.

17 Erkinjuntti-Pekkanen R, Rytkonen H, Kokkarinen JI, et al. Long-term risk of emphysema in patients with farmer's lung and matched control farmers. Am J Respir Crit Care Med 1998; 158: 662-665.

18 Okamoto T, Miyazaki Y, Ogura T, et al. Nationwide epidemiological survey of chronic hypersensitivity pneumonitis in Japan. Respir Investig 2013; 51: 191-199.

19 Wang L-J, Cai H-R, Xiao Y-L, et al. Clinical characteristics and outcomes of hypersensitivity pneumonitis: a population-based study in China. Chin Med J 2019; 132: 1283-1292.

20 Adegunsoye A, Oldham JM, Demchuk C, et al. Predictors of survival in coexistent hypersensitivity pneumonitis with autoimmune features. Respir Med 2016; 114: 53-60.

21 Ojanguren I, Morell F, Ramón MA, et al. Long-term outcomes in chronic hypersensitivity pneumonitis. Allergy 2019; 74: 944-952.

22 Jacob J, Odink A, Brun AL, et al. Functional associations of pleuroparenchymal fibroelastosis and emphysema with hypersensitivity pneumonitis. Respir Med 2018; 138: 95-101.

23 Morell F, Roger A, Reyes L, et al. Bird fancier's lung: a series of 86 patients. Medicine (Baltimore) 2008; 87: 110-130.

24 Ley B, Newton CA, Arnould I, et al. The MUC5B promoter polymorphism and telomere length in patients with chronic hypersensitivity pneumonitis: an observational cohort-control study. Lancet Respir Med 2017; 5: 639-647.

25 Gimenez A, Storrer K, Kuranishi L, et al. Change in FVC and survival in chronic fibrotic hypersensitivity pneumonitis. Thorax 2018; 73: 391-392.

26 Perez-Padilla R, Salas J, Chapela R, et al. Mortality in Mexican patients with chronic pigeon breeder's lung compared with those with usual interstitial pneumonia. Am Rev Respir Dis 1993; 148: 49-53.

27 Lima MS, Coletta EN, Ferreira RG, et al. Subacute and chronic hypersensitivity pneumonitis: histopathological patterns and survival. Respir Med 2009; 103: 508-515.

28 De Sadeleer L, Hermans F, De Dycker E, et al. Effects of corticosteroid treatment and antigen avoidance in a large hypersensitivity pneumonitis cohort: a single-centre cohort study. J Clin Med 2018; 8: 14.

29 Mooney JJ, Elicker BM, Urbania TH, et al. Radiographic fibrosis score predicts survival in hypersensitivity pneumonitis. Chest 2013; 144: 586-592.

30 Hanak V, Golbin JM, Hartman TE, et al. High-resolution CT findings of parenchymal fibrosis correlate with prognosis in hypersensitivity pneumonitis. Chest 2008; 134: 133-138.

31 Vourlekis JS, Schwarz MI, Cherniack RM, et al. The effect of pulmonary fibrosis on survival in patients with hypersensitivity pneumonitis. Am J Med 2004; 116: 662-668.

32 Adams TN, Newton CA, Glazer CS. Role of antigen type in survival in chronic hypersensitivity pneumonitis Lung 2019; 197: 113-114.

33 Tsutsui T, Miyazaki Y, Kuramochi J, et al. The amount of avian antigen in household dust predicts the prognosis of chronic bird-related hypersensitivity pneumonitis. Ann Am Thorac Soc 2015; 12: 1013-1021.

34 Sema M, Miyazaki Y, Tsutsui T, et al. Environmental levels of avian antigen are relevant to the progression of chronic hypersensitivity pneumonitis during antigen avoidance. Immunity Inflamm Dis 2018; 6: 154-162.

35 Monkare S, Haahtela T. Farmer's lung - a 5-year follow-up of eighty-six patients. Clin Allergy 1987; 17: 143-151.

36 Bourke SJ, Banham SW, Carter R, et al. Longitudinal course of extrinsic allergic alveolitis in pigeon breeders. Thorax 1989; 44: 415-418.

37 Ohtsuka Y, Munakata M, Tanimura K, et al. Smoking promotes insidious and chronic farmer's lung disease, and deteriorates the clinical outcome. Intern Med 1995; 34: 966-971.

38 Warren CP. Extrinsic allergic alveolitis: a disease commoner in non-smokers. Thorax 1977; 32: $567-569$.

39 Raghu G, Amatto VC, Behr J, et al. Comorbidities in idiopathic pulmonary fibrosis patients: a systematic literature review. Eur Respir J 2015; 46: 1113-1130.

40 Koschel DS, Cardoso C, Wiedemann B, et al. Pulmonary hypertension in chronic hypersensitivity pneumonitis Lung 2012; 190: 295-302.

41 Nadrous HF, Pellikka PA, Krowka MJ, et al. Pulmonary hypertension in patients with idiopathic pulmonary fibrosis. Chest 2005; 128: 2393-2399.

42 Cottin V, Nunes H, Brillet PY, et al. Combined pulmonary fibrosis and emphysema: a distinct underrecognised entity. Eur Respir J 2005; 26: 586-593.

43 Travis WD, Costabel U, Hansell DM, et al. An official American Thoracic Society/European Respiratory Society statement: update of the international multidisciplinary classification of the idiopathic interstitial pneumonias. Am J Respir Crit Care Med 2013; 188: 733-748.

44 Reddy TL, Tominaga M, Hansell DM, et al. Pleuroparenchymal fibroelastosis: a spectrum of histopathological and imaging phenotypes. Eur Respir J 2012; 40: 377-385. 
45 Jacob J, Bartholmai BJ, Egashira R, et al. Chronic hypersensitivity pneumonitis: identification of key prognostic determinants using automated CT analysis. BMC Pulm Med 2017; 17: 81.

46 Chung JH, Montner SM, Adegunsoye A, et al. CT findings associated with survival in chronic hypersensitivity pneumonitis. Eur Radiol 2017; 27: 5127-5135.

47 Chung JH, Zhan X, Cao M, et al. Presence of air trapping and mosaic attenuation on chest computed tomography predicts survival in chronic hypersensitivity pneumonitis. Ann Am Thorac Soc 2017; 14: 1533-1538.

48 Salisbury ML, Gu T, Murray S, et al. Hypersensitivity pneumonitis: radiologic phenotypes are associated with distinct survival time and pulmonary function trajectory. Chest 2019; 155: 699-711.

49 Walsh SLF, Sverzellati N, Devaraj A, et al. Chronic hypersensitivity pneumonitis: high resolution computed tomography patterns and pulmonary function indices as prognostic determinants. Eur Radiol 2012; 22: $1672-1679$.

50 Churg A, Sin DD, Everett D, et al. Pathologic patterns and survival in chronic hypersensitivity pneumonitis. Am $J$ Surg Pathol 2009; 33: 1765-1770.

51 Chiba S, Tsuchiya K, Akashi T, et al. Chronic hypersensitivity pneumonitis with a usual interstitial pneumonia-like pattern: correlation between histopathologic and clinical findings. Chest 2016; 149: $1473-1481$.

52 Ohtani Y, Saiki S, Kitaichi M, et al. Chronic bird fancier's lung: histopathological and clinical correlation. An application of the 2002 ATS/ERS consensus classification of the idiopathic interstitial pneumonias. Thorax 2005; 60: 665-671.

53 Gaxiola M, Buendía-Roldán I, Mejía M, et al. Morphologic diversity of chronic pigeon breeder's disease: clinica features and survival. Respir Med 2011; 105: 608-614.

54 Hutchinson JP, McKeever TM, Fogarty AW, et al. Surgical lung biopsy for the diagnosis of interstitial lung disease in England: 1997-2008. Eur Respir J 2016; 48: 1453-1461.

55 Peljto AL, Zhang Y, Fingerlin TE, et al. Association between the MUC5B promoter polymorphism and survival in patients with idiopathic pulmonary fibrosis. JAMA 2013; 309: 2232-2239.

56 Noth I, Zhang Y, Ma SF, et al. Genetic variants associated with idiopathic pulmonary fibrosis susceptibility and mortality: a genome-wide association study. Lancet Respir Med 2013; 1: 309-317.

57 Ley B, Torgerson DG, Oldham JM, et al. Rare protein-altering telomere-related gene variants in patients with chronic hypersensitivity pneumonitis. Am J Respir Crit Care Med 2019; 200: 1154-1163.

58 Collard HR, Ryerson CJ, Corte TJ, et al. Acute exacerbation of idiopathic pulmonary fibrosis. an international working group report. Am J Respir Crit Care Med 2016; 194: 265-275.

59 Song JW, Hong S-B, Lim C-M, et al. Acute exacerbation of idiopathic pulmonary fibrosis: incidence, risk factors and outcome. Eur Respir J 2011; 37: 356-363.

60 Kolb M, Bondue B, Pesci A, et al. Acute exacerbations of progressive-fibrosing interstitial lung diseases. Eur Respir Rev 2018; 27: 180071 .

61 Olson AL, Huie TJ, Groshong SD, et al. Acute exacerbations of fibrotic hypersensitivity pneumonitis: a case series. Chest 2008; 134: 844-850.

62 Miyazaki Y, Tateishi T, Akashi T, et al. Clinical predictors and histologic appearance of acute exacerbations in chronic hypersensitivity pneumonitis. Chest 2008; 134: 1265-1270.

63 Miyazaki Y, Unoura K, Tateishi T, et al. Higher serum CCL17 may be a promising predictor of acute exacerbations in chronic hypersensitivity pneumonitis. Respir Res 2013; 14: 57.

64 Maher TM, Oballa E, Simpson JK, et al. An epithelial biomarker signature for idiopathic pulmonary fibrosis: an analysis from the multicentre PROFILE cohort study. Lancet Respir Med 2017; 5: 946-955.

65 Raghu G, Remy-Jardin M, Myers JL, et al. Diagnosis of idiopathic pulmonary fibrosis. An Official ATS/ERS/JRS/ ALAT Clinical Practice Guideline. Am J Respir Crit Care Med 2018; 198: e44-e68.

66 Nukui Y, Miyazaki Y, Masuo M, et al. Periostin as a predictor of prognosis in chronic bird-related hypersensitivity pneumonitis. Allergol Int 2019; 68: 363-369.

67 Long X, He X, Ohshimo S, et al. Serum YKL-40 as predictor of outcome in hypersensitivity pneumonitis. Eur Respir J 2017; 49: 1501924.

68 Janssen R, Grutters JC, Sato H, et al. Analysis of KL-6 and SP-D as disease markers in bird fancier's lung. Sarcoidosis Vasc Diffus Lung Dis 2005; 22: 51-57.

69 Sterclova M, Smetakova M, Stehlik L, et al. Bronchoalveolar lavage cell profiles and proteins concentrations can be used to phenotype extrinsic allergic alveolitis patients. Multidiscip Respir Med 2019; 14: 13.

70 Jacob J, Bartholmai BJ, Rajagopalan S, et al. Automated computer-based CT stratification as a predictor of outcome in hypersensitivity pneumonitis. Eur Radiol 2017; 27: 3635-3646.

71 Sahin H, Brown KK, Curran-Everett D, et al. Chronic hypersensitivity pneumonitis: CT features comparison with pathologic evidence of fibrosis and survival. Radiology 2007; 244: 591-598.

72 Yoshizawa Y, Miyashita Y, Inoue T, et al. Sequential evaluation of clinical and immunological findings in hypersensitivity pneumonitis: serial subclass distribution of antibodies. Clin Immunol Immunopathol 1994; 73: 330-337.

73 Adegunsoye A, Oldham JM, Chung JH, et al. Phenotypic clusters predict outcomes in a longitudinal interstitial lung disease cohort. Chest 2018; 153: 349-360.

74 Flaherty KR, Wells AU, Cottin V, et al. Nintedanib in progressive fibrosing interstitial lung diseases. $N$ Engl J Med 2019; 381: 1718-1727.

75 Khan F, Stewart I, Howard L, et al. The Its Not JUST Idiopathic pulmonary fibrosis Study (INJUSTIS): description of the protocol for a multicentre prospective observational cohort study identifying biomarkers of progressive fibrotic lung disease. BMJ Open Respir Res 2019; 6: e000439. 